\section{Negative Space Theory}

Jianping Xue

(Wuxi 214000,Jiangsu,China)

\section{jpxue@sina.com}

Abstract: It is necessary to continue the research on the formation mechanism of gravitation, the formation mechanism of black hole, the formation mechanism of mass, and the mechanism of symmetry breaking. This study is dedicated to establish a new theory, and compare this new theory with the existing theory, testing self-consistency and rationality of this new theory. Based on the observed physical facts, the negative space theory is be put forward according to the principle of scale conservation and spatial discontinuity, that is, space expansion produces positive space and Huazi, and there is a trap field around Huazi. Comparison results show that the negative space theory can better reveal the above physical mechanism, and changed the previous understanding of these physical mechanism, open up a new research direction.

Key words: negative space; trap field; gravity; Huazi

\section{Introduction}

Newton discovered the law of gravitation, but he didn't know why there is gravitation between mass, and what causes the gravitation. Einstein established the generalized relativistic gravitational field equation, that gravitation is the space-time bending due to the distribution of matter energy momentum, but the reason why matter cause space-time bending not be explained. For the black hole of the singularity, so far can not give a reasonable physical meaning.

The quality energy equation established by Einstein, indicating that the quality and energy can be transformed from each other, but there is no mechanism to reveal how energy is converted into quality. Higgs proposed the Higgs field and its mechanism, that the matter quality is the result of the particle's action in the Higgs field, but the source of the Higgs field is not explained, only to say that the universe is full of the Higgs field everywhere.

The first impetus of symmetry broken has not been found so far, and have to call it as "spontaneous". In addition, it is generally felt that the theory of general relativity and quantum mechanics is difficult to integrate. All these problems not only have been puzzling, but also hindered the further development of physics, which must be resolved.

A large number of observations confirm that the space is so large that it can only be measured in light years as a unit of length. Although there is such a huge space exists, but it gives a feeling of empty, and is difficult to be studied. Even if Dirac think of vacuum as a energy sea, but only think it as a container, from where virtual pair particles haunt.

From a large number of existing research results, a neglected key factor be found, that is the product of space expansion. Through the 
relationship between energy, quality and space , recognize the existence of negative space, then proposed the negative space theory.

\section{Negative space theory}

Astronomer Hubble found that there is a redshift in the cosmic galaxies, thus establishing Hubble's law and determining that the space is constantly expanding. This event reveals two characteristics: the space is expandable, and the expansion takes place inside the universe space.

Define the original scale $R_{0}$, scale after expansion $R_{1}$, expansion scale $\Delta R$, then:

Before space expansion, the original scale can be written as:

$$
R_{0}=R_{0}+0
$$

If space expand, the expansion scale $\Delta R$ is the difference between the scale after expansion and the original scale:

$$
\Delta R=R_{1}-R_{0}
$$

Then the scale after expansion can be written as:

$$
R_{1}=R_{0}+\Delta R
$$

Compare (1) and (2), the expansion scale $\Delta R$ corresponds to the 0 scale, that is to say the expansion mode of space must be a kind of expansion which something born from nothing, so expansion increment $\Delta R$ is obtained from the space of0 scale.

It can be learned that there is 0 volume space in the space, and the spatial increment can be obtained from this 0 volume space by expansion.

Propose the following principles:

Principle 1, the scale follows the principle of conservation, that is, the total scale remains unchanged when the scale change.

Scale has length, area and volume, define the following dimensions: length $L$, positive length $L^{+}$, negative length $L^{-}$;area $S$, positive area $S^{+}$, negative area $S$; volume $V$, positive volume $V^{+}$, negative volume $V^{-}$.

For a length region, its initial length $L$ is 0 , that is, there is no length or $L=0$. When the region is generated or expands, that is to say , the positive length $L^{+}$is generated, then:

$$
L=L^{+}+L^{-}
$$

$$
\begin{aligned}
& L=0 \\
& L^{-}=-L^{+}
\end{aligned}
$$

This indicates that when the length is generated, a negative length $L^{-}$corresponding to the positive length $L^{+}$will be obtained.

For an area region, its initial area $S$ is 0 , that is, there is no area or $S=0$. When the region is generated or expands, that is to say, the positive area $S^{+}$is generated, then:

$$
\begin{aligned}
& S=S^{+}+S^{-} \\
& S=0 \\
& S^{-}=-S^{+}
\end{aligned}
$$

This indicates that when the area is generated, a negative area $S^{-}$corresponding to 
the positive area $S^{+}$will be obtained.

For a spatial region, its initial volume $\mathrm{V}$ is 0 , that is, there is no volume or $V=0$. When the volume is generated or the space is inflated, that is to say, the positive volume $\mathrm{V}^{+}$is generated, then:

$$
\begin{aligned}
& V=V^{+}+V^{-} \\
& V=0 \\
& V^{-}=-V^{+}
\end{aligned}
$$

This indicates that when the space expands, a positive volume $V^{+}$and a negative volume $V^{-}$ corresponding to the positive volume will be obtained.

The above positive length $L^{+}$, positive area $\mathrm{S}^{+}$and positive volume $\mathrm{V}^{+}$are real worlds that people can feel, and corresponding to them, there are negative length $L^{-}$, negative area $S^{-}$ and a negative volume $V$.

Positive volume constitutes positive space, negative volume constitutes negative space.

Principle 2, the space is discontinuous and quantized.

Here the proof:

Let $\mathrm{O}$ as a point on one dimension line, $\mathrm{A}$ point and $B$ point is adjacent to $O$ point from right and left, and the distance between them is:

$$
\begin{aligned}
& \overline{A O}=\overline{O B}=0 \\
& \overline{A O B}=0 \\
& \overline{A B}=0
\end{aligned}
$$

That is to say , $A$ and $B$ is continuous to one another.

According to the continuous meaning, no matter in any case, one point can never be separated.

But according to the results observed by Hubble, that space can expand internal, that is to say something can be born from nothing, so expansion can occur within $O$ point, then:

$$
\overline{A O B}=\Delta \mathrm{L} \neq 0
$$

That mean A point, B point is not the same point, $\mathrm{A}$ and $\mathrm{B}$ can't be continuous, else the $\Delta L$ cannot be obtained, then:

$$
A \neq B
$$

That mean the space will be non-continuous space. From the form of spatial expansion, it can be seen that the positive space generated from the expansion of the 0 space points, and will make the space discontinuous. The non-continuous space will inevitably lead to the quantization of space, otherwise, non-quantized space must be a continuous space.

According to Principle 1 and Principle 2, when the cosmic space expands (or explodes), it will produce the positive volume and the negative volume from 0 volumes, then the positive volume and the negative volume are separated, and the positive volume forms the positive space, the positive space constitutes 
the universe space, the negative volume forms the negative space and curled into a negative space particle with no- volume in positive space, this particle will be called as Huazi. Huazi is the basic unit of matter material. Around the Huazi, there is a trap field between the Huazi and the positive space due to the change from positive to negative property. And the trap field have a role in relevant physical mechanism. This is the negative space theory.

Let's explain the reason of Hubble's redshift with this negative space theory.

The photon's energy is decided by it's wavelength (or frequency), when redshift occur, that mean the photon's wavelength becomes long, this gives the evidence of universe space expansion, and it is considered that the expansion of space elongates the wave of photons. Wavelength becomes long, that mean photon loses energy, but energy loses to where? No reasonable answer for this until now.

According to the negative space theory, the Huazi will be produced after the space expansion ,and it has a trap field. This trap field can absorb energy. When photon encounter this trap field, photon will lose energy, then photon's wavelength Increase, redshift occurs.

This shows that the cosmology redshift is not caused by the space expansion to elongate the photon's wavelength, but due to the loss of photon's energy by the huazi's trap field. As the space is constantly expanding, the more distant the photons from us, the more trap field for photons encounter on the way to the earth, will cause more energy loss and produce a greater redshift. This result consistent with the description of Hubble's law.

These lost energy will be converted into energy or dark energy and together with the Huazi to form matter or dark matter.

The theory of the Big Bang needs to solve the problem of how the outer and inner parts of the universe expand. The existing theories have membrane theory, parallel cosmology theory or multiple cosmology theory, but the membrane theory needs to assume that the outer periphery of the membrane is free to be used for expansion, Cosmology or multiple cosmology can't explain the expansion of the universe within the source, so these theories are unreasonable. According to the negative space theory, space expansion can be generated from 0 volume of the space, that is, in the universe and at the edge of the space, there can be 0 volume space expansion, without the need for free space for space expansion. Negative space theory solves the problem of the origin of space expansion.

It can be seen that the negative space theory can explain the phenomenon of cosmological redshift and spatial expansion reasonably, and has self-consistency. 
3 Analysis for relevant physical events with negative space theory

\subsection{The transformation of mass and energy}

Define energy $E$, mass $M$, speed of light $C$.

Einstein's energy equation is:

$E=M C^{2}$

From this equation it can be seen that the quality and energy have an equivalent relationship, which implies that the quality and energy can be transformed into each other.

Product of the positive matter and antimatter annihilation reaction is energy, that is, the matter disappear, all become energy. Annihilation reaction of positive and negative electron, generates a pair of photons, therefore, to admit photon as a pure energy quantum is reasonable.

The following analysis the conditions for energy into quality.

According to the Big Bang theory, 10-43 seconds after the Big Bang, the universe temperature is about $10^{32}$ degrees, the universe appears from the quantum fluctuations in the background, $10^{-35}$ seconds after the big bang, the universe temperature is about $10^{27}$ degrees, gravitation separated, quarks, bosons, leptons formed, $10^{-10}$ seconds after the Big Bang, the universe temperature is about $10^{15}$ degrees, protons and neutrons formed, 0.01 seconds after the Big Bang, the universe temperature is about 100 billion degrees, photons, electrons, neutrinos as the main, proton neutrons accounted for only one of a billion, the rapid expansion of the system, the temperature and density continue to decline [1-2].

The Big Bang caused a dramatic expansion of space, a sharp decline in the temperature of the universe, and the energy was cooled into material, which is the way described in the Big Bang theory that the energy is changed into material particles. But this conclusion is not correct.

First, the cosmic microwave background radiation shows that the universe background temperature is $3 \mathrm{k}$ now, this temperature has fallen hundreds of millions of times compared to the beginning of the universe. Human beings use physical information to explore billions or even ten billion light years distant galaxies, this physical information of these galaxies is in the form of energy quantum such as electromagnetic waves, and which has existed on the cold distance of "billion light years". According to the big bang theory, all energy quantum should has been cooled into material particles in very high temperature after the big bang, so should not be seen any energy quantum in so low temperature background now, but in fact, these energy quantum can still be found to run along the long distance without "cooling" frozen into the material to reach the Earth. 
Second, the sun continues to emit solar energy around it, and these solar energy is not cooled into substance in the $3 \mathrm{k}$ temperature background of the Universe, but these solar energy was frozen as substance in the sun before it emits energy.

The third, positive electron and negative electron annihilation reactions produce a pair of observable pure quantum (photons), which also indicates that the energy quantum can not be frozen into matter particles in the $3 \mathrm{k}$ temperature background of the Universe now .

In addition, there are a large number of energy fields in the universe, including energy fields such as electric fields, magnetic fields, gravitational fields, etc., and the presence of these fields also shows that the low temperature can not convert energy quantum into particles also.

Therefore, the above facts show that the temperature drop do not convert energy into quality.

As the energy quantum, obviously it will not automatically convert to quality by itself, must rely on external conditions. So, after excluding the temperature factor, the external conditions only have the space and the Higgs field, but whether the Higgs field can convert energy into quality will be discussed later.

According the negative space theory, the spatial expansion produce the positive space and Huazi.

From the relativistic quality formula and the energy equation, it is known that the energy quantum is usually run at the light speed, and the quality particles can not run at the light speed. Therefore, to convert the energy quantum into quality particles, it is necessary to change the state of the energy quantum, such as from the photon state to the electronic state, corresponding to its speed must also be reduced

At present, the temperature of the universe is relatively cold compared with the beginning of the universe, but the energy of light and electromagnetic waves is still in the "vacuum" in the speed of light transmission, from this objective fact, we can determine that the space has not the ability to transform energy into mass.

So, after excluding the temperature factor and the positive space factor, the condition for energy quantum to be transformed into mass, there only the Huazi and the Higgs field.

First analyze the role of the Huazi.

There is a trap field around the Huazi, which has the opposite physical effect to the positive space. Thus, when the energy quantum reaches the Huazi, it's state will change by the action of the trap field, and it's velocity will be reduced, like as the effect of "brake". Then the energy quantum converts into basic unit of 
material - the Gouzi, and has mass, see Figure 1. Here, the basic unit that converted from energy quantum is called the Gouzi.

In the process of this transformation, the "force" effect is exhibited. This force is generated by the "brake" action on the energy quantum by Huazi, so that the energy can be converted into mass. This force is the source of various forces, such as gravitation, electromagnetic force, strong interaction force, etc. The direction of the force is determined by the nature of the interaction of the energy quantum and the Huazi, see Figure 2. Under the action of the Huazi, the energy quantum is converted into the basic unit Gouzi and combined with the Huazi, see Figure 3. The Gouzi and the Huazi are combined into $\mathrm{GHzi}$ unit, see Figure 4.

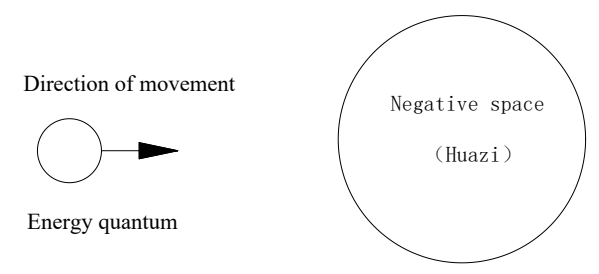

Figure 1 energy quantum and negative space (Huazi), the dotted circle is the trap field range of the negative space (Huazi) .

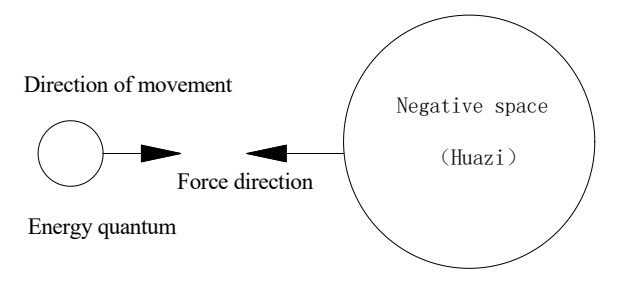

Figure 2 Negative space (Huazi) acts on the energy quantum, force exits

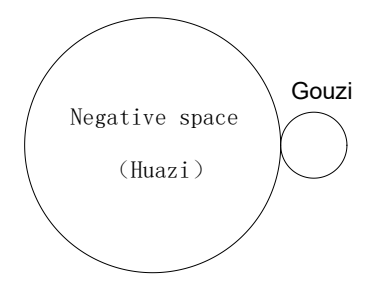

Figure 3 Negative space (Huazi) converts energy quantum into the Gouzi(element)

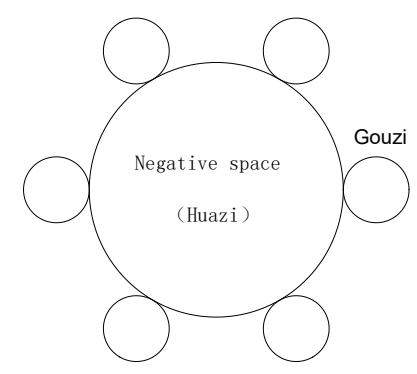

Figure 4 Negative space (Huazi) and Gouzi(element)

composite GHzi unit

It can be seen that the Huazi is space, the Gouzi is energy. The combination of the Huazi and the Gouzi is the organic combination of energy and space. The GHzi is the most basic unit of matter, and all the elementary particles are formed by the combination of the GHzi unit. The force is the concomitant product when the energy quantum is converted into material particles.

In 1923 the American physicist Compton in the study of graphite in the X-ray scattering of electrons found that some of the wavelengths of scattered waves are slightly larger than the wavelength of the incident wave, this phenomenon is called Compton effect.

The experimental result is:

(1) In the scattered light, in addition to the same spectral line as the original wavelength $\lambda_{0}$, a spectral line of a new wavelength $>\lambda_{0}$ is 
generated.

(2) The amount of change in wavelength $\Delta \lambda=\lambda-\lambda_{0}$ increases as the scattering angle $\varphi$ (the angle between the scattering direction and the incident direction) increases.

(3) For scattering materials of different elements, the amount of change $\Delta \lambda$ of the wavelength is same at the same scattering angle. The intensity of the scattered light with a wavelength of $\lambda$ decreases as the atomic number of the scattering materials' atoms increases.

According to the law of conservation of energy and momentum:

$$
\begin{aligned}
& \left\{\begin{array}{l}
h v_{0}+m_{0} c^{2}=h v+m c^{2} \\
\frac{h v_{0}}{c} n_{0}=\frac{h v}{c} n+m V
\end{array}\right. \\
& \lambda-\lambda_{0}=\Delta \lambda=\frac{h}{m_{0} c}(1-\cos \phi)
\end{aligned}
$$

Where $h$ is the Planck constant, $v_{0}, v$ is the frequency before and after the photon collision, $m_{0}, m$ is the static mass before and after the electron collision, and $C$ is the speed of light.

It can be seen from the equations (15)[3]of the Compton effect that the energy of a photon can be transmitted (divided) into $h\left(v_{0}-v\right)$, and one part of this divided energy $h\left(v_{0}-v\right)$ transmitted to the electron becomes the momentum $m$ Vof electron, the other part this divided energy $h\left(v_{0}-v\right)$ transmitted to the electron becomes mass $\left(m-m_{0}\right)$ of the electron.
The Compton effect experiment results show that photons can be divided into smaller photons, which are separable and can be converted into partial static mass of electrons. If the Higgs field exists everywhere according to the Higgs mechanism, the photon should become a mass particle under the action of the Higgs field, instead of becoming a mass particle after encountering the electron.

The Higgs mechanism is not true.

The Compton effect shows that according to equation (16), the change in wavelength can be a continuous change with the scattering angle $\varphi$, that is, the energy of the photon can be divided into smaller parts, up to the inseparable smallest micro-photons. Micro-photons can also be converted into micro-mass, that is, a photon can be subdivided into micro-photons, and micro-photons corresponding to micro-particles, indicating that the basic particles in the existing standard particle model are not basic. The elementary particles can still be composed of more basic particles. Therefore, the existing elementary particle model must be modified.

According to the negative space theory, due to the existence of electrons in the electronic, the potential of the potential field with absorption, conversion function. Thus, when a trap field is encountered, a single photon can be absorbed (divided) by a different amount of energy from the trap field with a different degree 
of action (incident angle $\varphi$ ) from the trap field and absorbed into the mass of electrons, In the transformation process to produce force, so that electrons gain momentum. This is in line with the Compton effect and its interpretation.

According to the negative space theory, due to the presence of the Huazi in the electron, the trap field of the Huazi has the function of absorption and transformation. Therefore, when a single photon encounters this trap field, it can be absorbed (divided) with a different degree of action (incident angle $\varphi$ ) by the trap field, and is absorbed into the mass of the electron, generating forces during the converting process, allowing the electrons to acquire momentum. This is consistent with the Compton effect and its interpretation.

It can be seen that the negative space theory can reasonably reveal the mechanism of energy conversion into mass.

\subsection{Source of quality}

British physicist P.W.Higgs proposed the Higgs mechanism. In this mechanism, the standard propagator and the fermion are given the mass by the Higgs field by acting with the Higgs field, which is the field quantization excitation of the Higgs field, which obtains the mass by self-interaction, All over the universe are filled with Higgs field.

Let's examine the process for electronic particle obtain mass.
According to the description of the Higgs mechanism, the electron first forms the electron shell, and it's mass is $M_{e 0}=0$, then the electronic shell acts in the Higgs field to obtain the electronic body's mass $M_{e 1}$, then:

Electronic mass $M_{e}$ is:

$$
M_{e}=M_{e}+M_{e 1}
$$

The electron energy $E_{e}$ is:

$$
E_{e}=M_{e 0} C^{2}+M_{e 1} C^{2}
$$

It can be seen from the equation (17) that the formation of the electron particle shell requires a source, according to the theory of quantum field, the particle shell $M_{\mathrm{e} 0}$ from the field excitation, the field is energy, so the shell has energy. At the same time, since the mass of the electronic body $M_{e 1}$ is given by the Higgs field (Higgs particle), according to the energy equation, the electronic body has no energy before the body quality $M_{e 1}$ is obtained.

It is observed that a whole electron all exists in mass state and not in pure energy state. Therefore, the shell $M_{\mathrm{e} o}$ must be converted into no zero mass state, that is, $M_{e 0} \neq 0$, but the mass of the shell is not given by the Higgs field.

This clearly shows that the Higgs mechanism is not true.

It is generally believed that $\mathrm{Y}$ photon with energy greater than $1.02 \mathrm{MeV}$ pass by a nuclei, the $y$ photon will be transformed into an electron and a positron, which is the positive and negative electron pair effect ofy photon and the 
typical energy conversion into matter. This phenomenon shows that the zero mass $\mathrm{Y}$ photon can be transformed into mass particles, secondly, the $y$ photon can occur a positive and negative electron pair effect only when it run close to the nucleus. In other words, the zero mass $\mathrm{Y}$ photon does not has a positive and negative electron pair effect in the spatial path before it encounters nucleus. According to the Higgs mechanism, the universe is full of Higgs field, which makes it possible for $y$ photon to transform into positive and negative electron before it encounters any nucleus. However, the fact proves that this is not the case, in addition, no experiments have confirmed that photon can be transformed into positive and negative electron in pure electromagnetic fields [4-5].

According to the negative space theory, it is the Huazi that converts energy into mass particles and gives them mass. Since the Huazi is present in the nucleus, when the high-energy $\mathrm{Y}$ photon encounters the nucleus or other particles which have Huazi, it will be affected by the trap field, then transform into a positive and negative electron.

It is predicted that in the ordinary pure electromagnetic field, even if the energy value of the high-energy $\mathrm{Y}$ photon reaches to the mass value of positive and negative electron, it is impossible to produce positive and negative electron because there is no Huazi negative space, unless the energy field is high enough to stimulate the spatial expansion to produce negative space.

The above logical arguments show that the Higgs mechanism is unreasonable and its role should be excluded.

It is more reasonable for the negative space theory to explain these above facts.

According to the negative space theory, it can will be predicted that in the area where the positive and negative electrons are annihilated, after the photon leaves, the Huazi will be left, and if there an energy quantum pass, there will occur new particles.

\subsection{Gravitational field equation}

Einstein established the gravitational field equation based on the relationship between matter and space [6], namely:

$$
R_{\mu \nu}-\frac{1}{2} g_{\mu v} R=\frac{8 \pi G}{c^{4}} T_{\mu \nu}
$$

Where $R_{\mu v}$ is the second order curvature tensor, $R$ is the curvature scalar, $g_{\mu v}$ is the gauge tensor, $T_{\mu v}$ is the energy and momentum tensor of the material, $G$ is the gravitational constant, and $C$ is the speed of light.

The left side of the equation (19) is the temporal and spatial geometry of the gravitational field, and the right is the energy momentum tensor of the material as the gravitational field source.

In the Schwarzschild coordinates (ct, r, $\theta, \varphi$ ) 
under the gravitational field equation, we can get Schwartz external solution, that is:

$d_{S}{ }^{2}=-\left(1-\frac{r_{s}}{r}\right) c^{2} d t^{2}+\left(1-\frac{r_{s}}{r}\right)^{-1} d r^{2}+r^{2}\left(d \theta^{2}+\sin ^{2} \theta d \varphi^{2}\right)$

Where $r_{s}=2 G M / c^{2}, \quad M$ is the mass.

From the photon motion with zero space-time element $d_{s}=0$ characteristics, photon "radial" movement, there are

$$
-\left(1-\frac{r_{s}}{r}\right) c^{2} d t^{2}+\left(1-\frac{r_{s}}{r}\right)^{-1} d r^{2}=0
$$

The coordinate velocity $V_{00}$ of the photon moving along the "radial" motion is:

$$
V_{00}=\left(1-\frac{r_{s}}{r}\right) c
$$

In (22), when $r=0$ and $r=r_{\mathrm{s}}, V_{00}$ result are singular, the latter $r=r_{\mathrm{s}}$ can be eliminated by selecting a coordinate system, while the former is inherent in space-time and is intrinsic[7-10].

Obviously, it is difficult to understand the physical meaning of $r=0$ before we realize the negative space, so we can only call it intrinsic. It can be known from the negative space theory that when $r=0$, it means that when the black hole formed, everything (at the radius of 0 ), is surrounded by the trapped field, and the external (positive space) is characterized by the trap field, that is, there has a strong negative space characteristic, as spatial singularity. That is to say, even if the positive space radius is 0 , the energy can still be allowed to exist there, which is present in the trapped field of the negative space.

According to Einstein's gravitational field equation, the gravitational force is the result of spatial distortion, which requires space to be a continuous space. Otherwise, the discontinuous space cannot be distorted, as it cannot twist a pile of sand.

However, the existence of matter particles is space-consuming, that is to say, space cannot be continuous, otherwise material particles cannot occupy space. Or, if the space is continuous, when the material particles move, the space recovery speed is different in the three dimensional directions (1 moving direction, 2 perpendicular to the moving direction) for the space left by the particles. The void area with no space will appear, thus destroying the continuity of the space, thus indicating that the space must be discontinuous. Therefore, it is not correct to treat space as continuous. It is not true that Einstein's general relativity equation describe gravity as a spatial distortion effect. The conclusion that mass causes space bending is not true.

According to the negative space theory, due to the presence of Huazi in the mass particles, the Huazi has a trap field. The effect of this field on the space around the mass particle is equivalent to the spatial distortion, which is the spatial distortion described in the gravitational field equation. 
Thus, Einstein's gravitational field equation is not a relational equation that determines the spatial characteristics by matter mass, but a spatial characteristic equation between Huazi and a positive space, that is to say, the trap field causes the space around the mass material to be distorted.

Negative space theory reveals the essential source of gravitational field and is more rational in connection with the internal composition of mass particles.

\subsection{Black hole}

According to the black hole theory, the black hole is caused by the gravitational collapse. This argument does not hold.

We examine the internal gravitational distribution of an ideal homogeneous solid sphere.

On the surface of an ideal solid sphere, according to the law of universal gravitation, the gravitational field strength (gravity acceleration) per unit mass is:

$$
g=K R
$$

Where $\mathrm{K}$ is the coefficient and $\mathrm{R}$ is the radius of the sphere. Obviously, at the center of the solid sphere, the gravitational force is zero, and the gravitational force of each point in the ball increases linearly with the distance from the center of the sphere. However, in a certain scale of the sphere, the gravitation is not greater than the strength of the atom, and it does not tear the atom and collapse. That is, it is impossible to "tightly compress" the material to form a black hole by gravitation, and it is impossible to generate a central singularity.

The Schwarzschild radius based on the general relativistic gravitational field equation has been used as the theoretical basis for studying the black hole.

Schwarzschild radius

$$
r_{s}=2 G M / C^{2}
$$

The gravitational field at the Schwarzschild radius

$$
g=G M / r_{S}^{2}
$$

Then

$$
\begin{aligned}
& g=C^{4} /(4 G M) \\
& M=C^{4} /(4 G g)
\end{aligned}
$$

Where $G$ is the gravitational constant, $M$ is the mass of the celestial body (black hole), and C is the speed of light.

Equation (27) shows that the mass of the black hole increases, the gravitational field strength at the Schwarzschild radius decreases, which is inconsistent with the greater the mass of the black hole and the stronger the gravitational field.

According to formula (27), the same black hole mass $M$ black has the gravitational field strength of the earth (or the sun) surface can be 
obtained, that is:

$$
M_{\text {黑 }}=C^{4} /\left(4 G g_{\text {地 }}\right)
$$

This shows that it is possible to find a black hole whose gravitational field strength at the Schwarz's radius is equal to the gravitational field strength (gravity acceleration) of the Earth's surface, but such gravitational field strength does not form a black hole on the earth, and the light can Escape, it can be said that the black hole does not exist.

Therefore, using the general relativity equation to explain the cause of black holes will cause contradictions.

According to the negative space theory, the cause of black holes can be explained in this way, that is, In greater matter, under interaction, the Huazis (negative space) in the material produce a chain reaction, which recombine to form large trap field. And constantly produce chain superposition effects to form black hole. The black hole's horizon is the trapping field and has a significant trapping effect on the photon, including trapping the photon in the trap field.

It can be seen that the negative space theory is reasonable. And it can be speculated that there are random black holes in the universe, and there are even "starved" black holes that are composed of a combination of Huazi without quality.

\subsection{Symmetry breaking}

The symmetry breaking is a major basic subject in the theory of quantum mechanics. However, for the origin of symmetry breaking, the first impetus for breaking is still not found, and the word "spontaneous" has been added for this purpose.

Former Soviet physicist A.D. Sakharov pointed out that the dynamics of Barryogenes is must meet three conditions

(1) There is an interaction that destroys the conservation of the number of baryons. Suppose the universe begins with a state where the number of baryons is zero. If there is no interaction that destroys the conservation of the number of baryons, then its baryon number will always remain zero.

(2) The interaction of the constitutive transformation (C) invariance and the charge conjugate-space inversion joint transformation (CP) invariant. Only in this way, the number of particles and anti-particles may not be equal, and thus it is possible to cause the asymmetry of the number of baryons and the number of anti-baryons.

(3) Deviation from heat balance. If the universe is in thermal equilibrium, then the CPT theorem knows that the average of the number of baryons will remain zero.

According to the previous discussion, there is a trap field around the Huazi, which is a field with a gradual change in spatial characteristics. 
It has the functions of compression, absorption, transformation, etc., and has a function of unidirectional action on physical quantities.

The $\mathrm{C}$ and $\mathrm{P}$ of the material particles are determined by the conversion from energy via Huazi. In the conversion of the energy into the material particle, in addition to imparting the mass to the material particle, the state of the energy is also modified. This includes changing the spin of the energy, the wave state, and the spatial form. After the modification, the particles exhibit charge characteristics such as positive charge, negative charge or no charge in addition to mass, spin.

The spatial characteristics of the Huazi and its trap field have the natural ability to destroy the conservation of the number of baryons and the invariance of $C$ and $C P$, and the masses reduce the energy density in the space after the energy is converted into mass particles, causing the thermal equilibrium to deviate. Therefore, the Huazi and the trapping field can satisfy the three conditions proposed by A.D. Sakharov.

The driving force of CP's spontaneous break is the result of the existence of the Huazi. Therefore, the negative space theory can reasonably explain the first driving force of $\mathrm{CP}$ breaking and the imbalance of positive and negative material quantities.

Negative space theory is reasonable.

\section{Conclusion}

According to the above analysis, it can be considered that the negative space exists objectively. It is the inevitable need and result of the expansion of the universe space. It has played a important role in the transformation of energy into matter, material mass formation, force generation, black hole formation and symmetry breaking.

According to the negative space theory, the particle mass is not obtained by the Higgs mechanism, but is transformed from energy by the action of the Huazi. There is no Higgs field and Higgs particle. The black hole and the gravitational field are the result of the Huazi and its trap field, not the result of mass. The driving force of symmetry breaking is from the Huazi and its trapping field. The basic particles of matter are from the combination of the $\mathrm{GHzi}$.

Based on the negative space theory, the following predictions can be made:

(1) The collision of a pair of high-energy photons does not produce positive and negative electrons.

(2) The Higgs mechanism does not exist, the Higgs particle does not exist, and the so-called Higgs particle is just a new particle or a new combination particle.

(3) The black holes in the universe are composed of Huazi. Therefore, there are countless black holes in the universe, there are black holes of various masses, and there is 
even a black hole whose quality does not correspond to the black hole horizon, that is, the "starved" black hole.

( 4 ) Gravitational waves are not the distortion of space.

These predictions will test the correctness of negative space theory.

\section{References}

[1] John Barrow. The origin of the universe [M].

Bian Yulin translation. Shanghai: Shanghai Science and Technology Press, 1995.

[2] Stephen Hawking. Time brief [M]. Xu Mingxian Wu Zhongchao translation. Hunan: Hunan Science and Technology Press, 2007.

[3] Chen Xiimou, Zhong Xihua. University Physics General Course Optical [M]. Beijing: University Press, 2011

[4] Han Lei. High energy photon generation of positive and negative electron pairs [J]. Jilin: Jilin University, 2012.

[5] Ren Na. Strong laser field in the positive and negative electron pairs [D]. Shanghai: East China Normal University, 2014.

[6] Yu Yunqiang. General theory of relativity [M]. Beijing: Peking University Press, 2000.

[7] Gao Xingru, Zhang Ning. Journal of Beijing Union University (Natural Science Edition), 2015,19 (2): 54-56 (in Chinese) [J]. Journal of Beijing Union University (Natural Science
Edition), 2015,19 (2): 54-56.

[8] Cai Ronggen, Cao Liming, Hu Yapeng. Black hole physics [J]. Modern Physics Knowledge, 2015, 27 (5): 16-25.

[9] symmetric Schwarzschild gravitational field to solve for the sense and the ball draw and analysis of black hole [J] University Physics, 2015, 34 (11): 25-27.

[10] Shao Dan. Space and time - from classic to quantum theory [M]. Hunan: Hubei Science and Technology Press, 2013. 